\section{Radiotherapy and hormone therapy in patients with high-risk prostate cancer}

Standard treatment of localized intermediate and/or high risk prostate cancer comprises external-beam radiotherapy (RT) and hormonal therapy. This combination improves overall survival, but there are still some controversies surrounding the value of pelvic lymph node irradiation, as well as the timing of androgen suppression. The Radiation Therapy Oncology Group (RTOG) study 94-13 was designed to investigate whether whole pelvic RT (WPRT) is better than prostateonly RT (PORT) for high-risk disease and how neoadjuvant hormone therapy compared with adjuvant hormone therapy.

The study comprised 1,292 patients with locally advanced adenocarcinoma of the prostate and a minimum of $15 \%$ lymph node involvement. There were four treatment arms: WPRT + NHT, WPRT + AHT, PORT + NHT, and $\mathrm{PORT}+\mathrm{AHT}$. This study revealed no statistically significant differences in overall survival; the only trend towards a difference in progressionfree survival was observed in the WPRT $+\mathrm{NHT}$ arm compared with the PORT + NHT and WPRT + AHT arms. There seems to have been an unexpected interaction between the timing of the hormone therapy and the scope of the radiation therapy.

Lawton et al. recommended further studies to find out whether this failure to demonstrate a significant advantage for WPRT + NHT compared to PORT + AHT is down to chance, or whether there is a biological explanation.

Original article Lawton CA et al. (2007) An update of the phase III trial comparing whole pelvic to prostate only radiotherapy and neoadjuvant to adjuvant total androgen suppression: updated analysis of RTOG 94-13, with emphasis on unexpected hormone/radiation interactions. Int J Radiat Oncol Biol Phys 69: 646-655

\section{Plasma HER2 and EGFR levels are prognostic factors in patients with prostate cancer}

Studies have shown that HER2 and EGFR expression are associated with disease progression in patients with metastatic or hormone-refractory prostate cancer. A study by Shariat et al. has examined whether the circulating levels of HER2 and EGFR are prognostic in patients with prostate cancer.

The study included 227 patients with clinically localized prostate cancer who were treated with radical prostatectomy and bilateral lymphadenectomy, and had not received any prior hormonal or radiation therapy. The median preoperative HER2 and EGFR levels were $10.0 \mathrm{ng} / \mathrm{ml}$ and $31.4 \mathrm{ng} / \mathrm{ml}$, respectively. Plasma HER2 levels were significantly elevated in patients with seminal vesicle invasion $(P=0.033)$ and high pathologic Gleason sum $(P=0.028)$. Multivariate Cox regression analyses adjusted for standard preoperative predictors showed that high HER2, low EGFR and high HER2:EGFR ratio were associated with $\mathrm{PSA}$ progression $(P<0.001, P=0.003$, and $P<0.001$, respectively). Multivariate Cox analyses adjusted for standard postoperative predictors showed that preoperative plasma EGFR levels and HER2: EGFR ratio were associated with PSA progression $(P=0.025$, and $P=0.007$, respectively). By contrast, no association was found between HER2 levels and PSA progression $(P=0.176)$. Patients with aggressive disease progression had higher preoperative HER2 levels and lower EGFR levels than did patients with nonaggressive disease progression $(P=0.023$, and $P=0.04$, respectively).

The authors conclude that preoperative plasma HER2 and EGFR levels provide prognostic information in patients with prostate cancer who have undergone radical prostatectomy.

Original article Shariat SF et al. (2007) Preoperative plasma HER2 and epidermal growth factor receptor for staging and prognostication in patients with clinically localized prostate cancer. Clin Cancer Res 13:5377-5384

\section{Prostatectomy confers the best chance of long-term prostate- cancer-specific survival}

The best method for managing localized prostate cancer is currently unclear, as randomized trials are yet to determine which strategy produces the best long-term outcome. In the absence of convincing trial data, in a population-based cohort study that was carefully controlled for confounding factors Merglen et al. have demonstrated that surgery confers the best chance of long-term prostate-cancer-specific survival. 